

\section{COLLEGE OF ENGINEERING \\ THE ENGINEERING EXPERIMENT STATION}

The Engineering Experiment Station was organized in 1909 as a part of the College of Engineering. The staff of the Station includes all members of the Faculty of the College of Engineering, together with Research Assistants supported by the Station Funds.

The Station is primarily an engineering research institution engaged in the investigation of fundamental engincering problems of general interest, in the improvement of engineering design, and in the development of new industrial processes.

The Station desires particularly to co-operate with industries of Missouri in the solution of such problems. For this purpose, there is available not only the special equipment belonging to the Station but all of the equipment and facilities of the College of Engineering not in immediate use for class instruction.

Inquiries regarding these matters should be addressed to

The Director,

Engineering Experiment Station

University of Missouri

Columbia, Missouri

\section{THE UNIVERSITY OF MISSOURI BULLETIN}

Published by the University of Missouri at Room 102, Building T-3, Columbia, Missourl. Entered as second-class maltex, January 2, 1914, at post office at Columbia, Missouri, under Aet of Congress of Augrat 24, 1912. Issued four times monthly October through May, three times monthly June through September. 


\title{
Stability of Laminar Flow in Curved Channels $\uparrow$
}

\author{
By Chia-Shun Yihł and W. M. Sangster@ \\ Lowa Institute of Hydraulic Research, State University of Iowa
}

[Received July 5, 1956]

\begin{abstract}
Occurrence of secondary flow of a viscous fluid flowing under a pressure gradient in curved channels has often been explained by invoking the existence of boundary layers before the primary flow is established. In this paper it is shown that instability can occur even after the full establishment of the primary flow. Chandrasekhar's method is used in the analysis and the relationship between the stability parameter and the wave number of the disturbance for neutral stability is obtained.
\end{abstract}

\section{§ 1. INTRODUCTION}

WhEREAs the occurrence of spirals in fluid flow under a pressure gradient in curved channels is common knowledge, explanations of the phenomenon have been based upon the existence of boundary layer's before the primary flow is fully established, and the fully developed two-dimensional flow has generally been believed to be free of such spirals (Goldstein 1938). In the present paper, this phenomenon will be analysed by Chandrasekhar's (1954) method under the assumption that the primary flow is two-dimensional and between two concentric circular cylinders of small spacing, and that it is already fully established. The object is to obtain definite information about the stability of such a flow against the formation of Taylor-Görtler vortices of various transverse wavelengths.

\section{§2. Velocity Distribution in the Primary Flow}

Cylindrical coordinates $(r, \phi, z)$ will be used, with the $z$-axis coinciding with the axis of the cylinders. If the radius of the inner cylinder is $a$ and that of the outer one is $b$, the distribution of the velocity $V$ of the primary flow can be found by solving the Navier-Stokes equations and is givell (Goldstein 1938) by

in which

$$
\frac{V}{K}=A\left(\frac{r}{b}-\frac{b}{r}\right)+\frac{r}{b} \ln \frac{r}{b} \quad . \quad . \quad . \quad . \quad . \quad .
$$

$$
A=\frac{a^{2}(\ln a-\ln b)}{b^{2}-a^{2}}, \quad K=\frac{b}{2 \mu} \frac{\partial P}{\partial \phi} \quad . \quad . \quad .
$$

$\mu$ being the clynamic viscosity and $P$ the pressure for the prinuary flow.

+ Communicated by S. Chandrasekhar, F.R.S.

+ Now at University of Michigan

$\S$ Now at University of Missuurj 
If the spacing $d$ of the cylinders (or $b-a$ ) is very small compared with the radii of the cylinders it can be shown from eqn. (1) that the velocity distribution of the primary flow is essentially the same as that for plane Poiseuille flow, i.e.

in which

$$
V=\frac{d^{2}}{2 \mu a^{2}} \frac{\partial P}{\partial \phi}\left(\xi^{2}-\xi\right)=\frac{K d^{2}}{a^{2}}\left(\xi^{2}-\xi\right) \quad . \quad . \quad .
$$

$$
\xi=\frac{r-a}{b} . \quad \text {. . . . . . . . . }
$$

The mean velocity is, to the same degree of approximation,

$$
V_{m}=-\frac{d^{2} K}{6 a^{2}}
$$

so that the Reynolds number based on $V_{m}$ and $d$ is

$$
R=\frac{V_{m} d}{\nu}=-\frac{d^{3} K}{6 \nu a^{2}} \quad \text {. . . . . . . }
$$

in which $\nu$ is the kinematic viscosity. From eqn. (3) it follows that, with terms of higher order in $d$ neglected,

$$
\begin{aligned}
& \frac{V}{r}=\frac{K d^{2}}{a^{3}}\left(\xi^{2}-\xi\right) \quad . \quad . \quad . \quad . \quad . \quad . \quad . . \\
& V^{\prime}+\frac{V}{r}=\frac{K d}{a^{2}}(2 \xi-1) \text {. . . . . . . }
\end{aligned}
$$

in which the prime indicates differentiation with respect to $r$. These quantities will be useful later in the investigation of stability.

\section{§ 3. Formulation of the Stability Problem}

To investigate the stability of the primary flow, one may superpose on it a time-dependent perturbation with (small) velocity components $(u, v, w)$ in the directions of $r, \phi$, and $z$, respectively, and with corresponding pressure $p$. If it is kept in mind that the primary flow is such that the Navier--Stokes equations and the equation of continuity are satisfied, and if terms of higher order than the first in the perturbation quantities are neglected, the Navier-Stokes equations are

$$
\begin{gathered}
\frac{\partial u}{\partial t}-\frac{2 V v}{r}=-\frac{1}{\rho} \frac{\partial p}{\partial r}+v\left(\nabla^{2} u-\frac{u}{r^{2}}\right), \\
\frac{\partial v}{\partial t}+u V^{\prime}+\frac{V u}{r}=v\left(\nabla^{2} v-\frac{v}{r^{2}}\right), \\
\frac{\partial w}{\partial t}=-\frac{1}{\rho} \frac{\partial p}{\partial z}+v \nabla^{2} w, \quad . \quad . \quad . \quad .
\end{gathered}
$$

in which $t$ and $\rho$ denote time and density respectively, and

$$
\nabla^{2} \equiv \frac{\partial^{2}}{\partial r^{2}}+\frac{1}{r} \frac{\partial}{\partial r}+\frac{\partial^{2}}{\partial z^{2}} .
$$


The equation of continuity is

$$
\frac{\partial(u r)}{\partial r}+\frac{\partial(w r)}{\partial z}=0 . \quad . \quad . \quad . \quad . \quad . \quad .
$$

Now, following Taylor (1923), one may assume

$$
(u, v, w)=\exp (\sigma t)\left[u_{1}(r) \cos k z, v_{1}(r) \cos k z, w_{1}(r) \sin k z\right] .
$$

If $p$ is eliminated between eqns. (8) and (10), and if eqn. (12) is used, one has

$$
\frac{v}{k} D\left(\nabla_{1}^{2}-k^{2}-\frac{\sigma}{\nu}\right) w_{1}=-\frac{2 V}{r} v_{1}-\nu\left(\nabla_{1}^{2}-k^{2}-\frac{1}{r^{2}}-\frac{\sigma}{\nu}\right) u_{1} .
$$

in which

$$
D \equiv d / d r, \quad \nabla_{1}^{2}=D^{2}+\frac{1}{r} D .
$$

Furthermore eqn. (9) can now be written as

$$
u_{1}\left(V^{\prime}+\frac{V}{r}\right)=v\left(\nabla_{1}^{2}-k^{2}-\frac{1}{r^{2}}-\frac{\sigma}{v}\right) v_{1} \quad . \quad .
$$

and the equation of continuity is

$$
\frac{u_{1}}{r}+D u_{1}+k w_{1}=0 .
$$

Although for neutral stability $\sigma$ may be purely imaginary, one may follow Taylor in taking it to be zero-at any rate if one is looking for the formation of Taylor-Görtler vortices. For neutral stability, therefore, eqn. (14) becomes

in which

$$
\nu L_{1} v_{1}=\left(V^{\prime}+V / r\right) u_{1} \quad . \quad . \quad . \quad . \quad . \quad .
$$

$$
L_{1} \equiv D^{2}+\frac{1}{r} D-k^{2}-\frac{1}{r^{2}}
$$

and it can be readily shown that eqns. (13) and (15) yield

$$
\frac{\nu}{k^{2}} L_{1}^{2} u_{1}=\frac{2 V}{r} v_{1} . \quad \text {. . . . . . . . . }
$$

Since the spacing $d$ is much smaller than $a$ or $b$, and since the perturbation velocities change appreciably as $r$ varies between $a$ and $b$, and the wavelength $(2 \pi / k)$ is comparable with $d$, the part

$$
\frac{1}{r} D-\frac{1}{r^{2}}
$$

in the operator $L_{1}$ is small compared with the rest of the terms. If now one denotes $k d$ by $\alpha$ and expresses $u_{1}$ and $v_{1}$ in terms of $V_{m}$, eqns. (16) and (17) become, in virtue of eqns. (5), (6), and (7),

$$
\begin{gathered}
L v_{1}=\frac{K d^{3}}{\nu a^{2}} u_{1}=-6 R(2 \xi-1) u_{1}, \quad . \quad . \quad . \\
L^{2} u_{1}=\frac{2 K k^{2} d^{6}}{\nu n^{3}}\left(\xi^{2}-\xi\right) v_{1}=-12 \alpha^{2} R \beta\left(\xi^{2}-\xi\right) v_{1} \quad . \quad .
\end{gathered}
$$


in which

If one sets

$$
L=d^{2} / d \xi^{2}-\alpha^{2}, \quad \beta=d / \alpha .
$$

one has finally

where

$$
\begin{aligned}
& u_{1}=-12 \alpha^{2} R \beta W \quad \text {. . . . . . } \\
& L v_{1}=S \alpha^{2}(2 \xi-1) W
\end{aligned}
$$

is the stability parameter. Equations (21) and (22) are to be solved with the boundary conditions

$$
v_{1}(0)=v_{1}(1)=0, \quad W(0)=W(1)=0, \quad W^{\prime}(0)=W^{\prime}(1)=0 .
$$

the last two being a consequence of eqns. (15) and (20) and of the fact that $u_{1}$ and $w_{1}$ are zero at $\xi=0$ and $\xi=1$. The primes in the last two of eqns. (24) indicate differentiation with respect to $\xi$. The differential system consisting of eqns. (21), (22), and (24) represents an eigenvalue problem, the solution of which will result in a functional relationship between $S$ and $\alpha$ for neutral stability since $\sigma$ has been assumed to be zero to start with.

\section{§4. Outline of the Mermod of Solution}

Since the method of solution used in this paper is that of Chandrasekhar and is already described in detail elsewhere (Chandrasekhar 1954), only an outline of the essential steps will be presented here. First, one takes

$$
v_{1}=\sum_{n=1}^{\infty} A_{n} \sin n \pi \xi . \quad \text {. . . . . . . . }
$$

to satisfy the first two conditions in eqn. (24). Then this expression for $v_{1}$ is substituted into eqn. (21) which can be solved to yield the result

$$
\begin{aligned}
W= & \sum_{m=1}^{\infty} \frac{A_{m}}{M^{2}}\left[B_{m} \cosh \alpha \xi+C_{m} \sinh \alpha \xi+D_{m} \xi \cosh \alpha \xi+E_{m} \xi \sinh \alpha \xi\right. \\
& \left.+\left(\xi^{2}-\xi\right) \sin m \pi \xi+\frac{4 m \pi(2 \xi-1) \cos m \pi \xi}{M}+\frac{4\left(M-6 m^{2} \pi^{2}\right) \sin m \pi \xi}{M^{2}}\right]
\end{aligned}
$$

in which $M=m^{2} \pi^{2}+\alpha^{2}$ and the constants $B$ to $D$ can be determined to satisfy the last four conditions in eqns. (24). With these constants so determined, eqn. (26) is then substituted into eqn. (22) and the right-liand side expressed in a Fourier sine series. Then coefficients of the individual harmonics at left and right are equated. This result in an infinite number of homogeneous linear equations involving the $A$ 's. Finally the eliminant of these equations (or the condition that the $A$ 's are not all zero) furnishes the desired relationship between $S$ and $\alpha$.

Although the eliminant is a determinant of infinitely many rows and columns, Chandrasekhar (1954) has shown that often it is sufficiently 
accurate to equate the first element (in the first column and first row) to zero, and that the result of the second approximation (by equating a two-by-two determinant to zero) does not differ much from that of the first. The same was found to be true in a previous calculation (for a slightly different primary flow) by the authors whenever the second approximation was tried for spot checking, so that the relationship between $S$ and $\alpha$ determined by the first approximation is believed to be sufficiently accurate. The ease with which this relationship was obtained is additional proof of the power of Chandrasekhar's method in solving problems of the present type.

\section{§5. Results and Concluding Remarks}

The desired relationship between $S$ and $\alpha$ for neutral stability of the primary flow is given graphically in the figure. It is seen from this

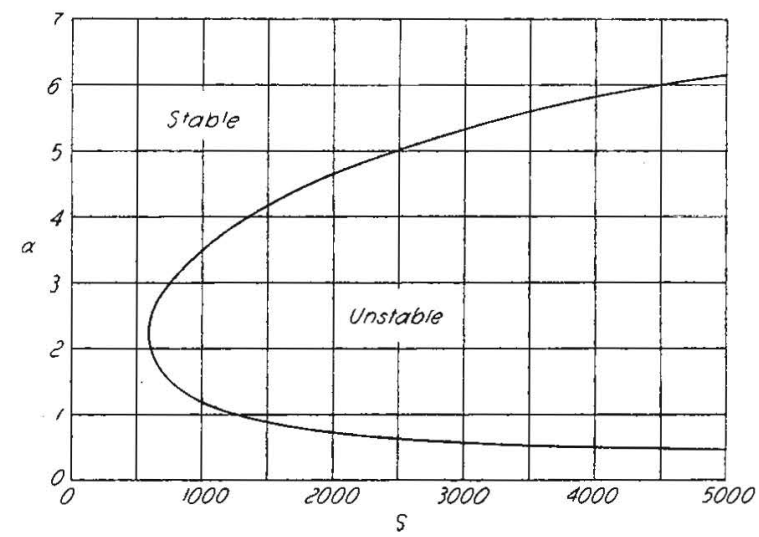

Relationship between the wave number $\alpha$ and the stability parameter $S$ for neutral stability.

figure that established laminar flow under a pressure gradient in a narrow curved channel is unstable for values of the parameter $S$ greater than the approximate critical value 600 , which corresponds to a wavelength of the Taylor-Görtler vortices equal to $2 \pi / 2 \cdot 25$ or 2.79 times the width of the channel. Since the flow tends to be unstable as $S$ is increased, for the same Reynolds number and the same (small) width of the channel it does so as the curvature is increased, as can be seen from the definition of $S$. Thus centrifugal force is as much a cause of instability for the established flow as it is for the unestablished one, and the occurrence of secondary flow cannot be considered to be exclusively a consequence of boundarylayer development.

Attention might also be called to the fact that, since centrifugal force call be considered as a form of gravitation (Mach, Einstein), the nonuniformity of it is a kind of stratification-a stratification not of density, for the density is homogeneous, but of specific weight in the general 
sense. Secondary flow occurs if this stratification is not stable, and the mechanics of it is not unlike the falling of a heavier fluid in a lighter one, particularly if the boundary conditions are alike, and diffusion takes place to render the variation of density and that of centrifugal acceleration strictly comparable. This point of view is substantiated elsewhere by the equality of the Rayleigh number for thermal instability and the Taylor number for rotational instability (both being 1708), as first pointed out by Low (1929).

\section{ACKNOWLEDGMENT}

This work was jointly sponsored by the Iowa Institute of Hydraulic Research and the Office of Ordnance Research under Contract number DA-11-022-ORD-1729.

\section{REFERENCES}

Goldstein, S. (Editor), 1938, Modern Developments in Fluid Dynamics (Oxford), vol. I, pp. 84-87, 315-316.

ChaNdRasekHaR, S., 1954, Mathematika, 1, 5.

TAylor, Sir Geoffrey, 1923, Phil. Trans. Roy. Soc. A, 223, 289.

Low, A. R., 1929, Proc. Roy. Soc. A, 125, 180. 




\section{PUBLICATIONS OF THE ENGINEERING REPRINT SERIES}

Copies of the complete list of publications may be secured from the Director of the Engineering Experiment Station, University of Missouri

Reprint No.

14. Selection of Personnel by George W. Elliott, Assistant Professor of Mechanical Engineer ing. Reprinted from the 1954 Transcript of the Midwest Feed Production School.

15. Lightweight Aggregates for Structural Concrete by Adrian Pauw, Associate Professor of Civil Engineering. Reprinted from the Proceedings of the A.S.C.E., Vol. 81, Separate No. 584, January 1955.

16. Coating Thickness Measurements Using Pulsed Eddy Currents by Donald L. Waidelich, Associate Director, Engineering Experiment Station. Reprinted from the Proceedings of the National Electronics Conference, Vol. 10, February 1955.

17. Additions to Sample-Data Theory by G. V. Lago, Associate Professor of Electrical Engineering. Reprinted from the Proceedings of the National Electronics Conference, Vol. 10, February 1955.

18. Additions to Z-Transformation Theory for Sample-Data Systems by Gladwyn V. Lago, Associate Professor of Electrical Engineering. Reprinted from Transactions of the American Institute of Electrical Engineers, Vol. 74, January, 1955.

19. Tension Control for High Strength Structural Bolts by Adrian Pauw, Professor of Civil Engineering and Leonard L. Howard, Lakeland Engineering Associates, Inc., with a discussion on the Turn-of-the-Nut Method by E. J. Ruble, Association of American Railroads. Reprinted from the Proceedings of the American Institute of Steel Construction, National Engineering Conference, April 18-19, 1955.

20. Autotransformer Betters Motor Phase Conversion by Joseph C. Hogan, Associate Professor of Electrical Engineering. Reprinted from Electrical World, Vol. 144, p. 120, October 17, 1955.

21. Sequence Summation Factors by Adrian Pauw, Professor of Civil Engineering. Reprinted from the Proceedings of the American Soc iety of Civil Engineers. Vol. 81, Paper No. 763, August, 1955.

22. Pulsed Eddy Currents Gage Plating Thickness by Donald L. Waidelich, Assoc iate Director, Engineering Experiment Station. Reprinted from Electronics, Vol. 28, p. 146, November, 1955.

23. Relay Protection for Lines Being Sleet-Melted by the Short-Circuit Method by J. C. Hogan, Associate Professor of Electrical Engineering and C. G. Pebler, Commonwealth Associates, Inc. Reprinted from Transactions of the American Institute of Electrical Engineers, Vol. 74, December, 1955.

24. Supplemental Irrigation....Careful Planning is Essential by Harry Rubey, Professor of Civil Engineering. Reprinted from What's New in Crops and Soils, Vol. 7, August-September, 1955.

25. Analysis of Single-Phase-to-Three-Phase Static Phase Converters by J. C. Hogan, Associate Professor of Electrical Engineering. Reprinted from Transactions of the American Institute of Electrical Engineers, Vol. 74, p. 403, January, 1956.

26. Enrollment and Incomes in Civil Engineering can be Increased by Harry Rubey, Professor of Civil Engineering. Reprinted from Journal of Engineering Education, Vol. 46, p. 236, November, 1955.

27. A Synthesis Procedure for Sampled-Data Systems by G. V. Lago, Associate Professor of Electrical Engineering. Reprinted from Proceedings of the National Electronics Conference, Vol. 11, p. 251, 1955.

28. Design of Optimum Phase-Shift Oscillators by Donald L. Waidelich, Associate Director, Engineering Experiment Station. Reprinted from Proceedings of the National Electronics Conference, Vol, 11, p. 222, 1955. This article also appeared in Electronics Equipment, Vol. 4, p. 38, April, 1956.

29. Investigation Concerning Polarization in Barium Titanate Ceramics by G. W. Marks, U. S. Navy Electronics Laboratory, Donald L. Waidelich, Associate Director Engineering Experiment Station, University of Missouri and L. A. Monson, U. S. Navy Electronics Laboratory. Reprinted from Transactions of the American Institute of Electrical Engineers, Vol. 75, Part I, p 469, 1956.

30. The Influence of Shank Area on the Tensile Impact Strength of Bolts by John Love, Jr., General Electric Company and O. A. Pringle, Associate Professor of Mechanical Engineering. Reprinted from Transactions of the American Society of Mechanical Engineers, Vol. 78, p 1489, October, 1956.

31. Measurement of Coating Thicknesses by Use of Pulsed Eddy Currents by Donald L. Waidelich, Associate Director, Engineering Experiment Station. Reprinted from Nondestructive Testing, Vol. 14, p 14, MayJune 1956.

32. Head Losses in Storm Drain Junction Boxes by Horace W. Wood, Professor of Civil Engineering. Reprinted from Highway Research Board Proceedings, Vol. 35, p 177, 1956.

33. Stability of Laminar Flow in Curved Channels by Chia-Shun Yih, Associate Professor of Engineering Mechanics, University of Michigan and W. M. Sangster, Associate Professor of Civil Engineering, University of Missouri. Reprinted from The Philosophical Magazine, Volume 2, Eighth Series, Page 305, March 1957.

*Out of Print. 

University of Missouri Libraries

University of Missouri

MU Engineering Experiment Station Series

Local Identifier

YihSangster1957

Capture information
Date captured
2018 May
Scanner manufacturer Ricoh
Scanner model
MP C4503
Scanning software
Optical resolution
$600 \mathrm{dpi}$
Color settings
File types
Grayscale, 8 bit; Color, 24 bit Tiff

Source information

Format

Content type

Notes
Book

Text

Digitized duplicate copy not retained in collection.

Derivatives - Access copy

Compression

Editing software

Resolution

Color

File types

Notes
LZW

Adobe Photoshop

$600 \mathrm{dpi}$

Grayscale, 8 bit; Color, 24 bit

Tiffs converted to pdf

Greyscale pages cropped and canvassed. Noise removed from background and text darkened. Color pages cropped. 